\title{
Remote Terrestrial Sites as Operational/Logistics Analogs for Moon/Mars Bases: the Haughton Mars Project
}

\author{
Erica Gralla, ${ }^{*}$ Sarah Shull, ${ }^{\dagger}$ Jaemyung Ahn, ${ }^{*}$ Afreen Sidiqqi,${ }^{\S}$ Olivier de Weck ${ }^{* *}$ \\ Department of Aeronautics \& Astronautics, Massachusetts Institute of Technology \\ Cambridge, MA 01239
}

\begin{abstract}
Mission planners for future human space exploration enterprises face several challenges in the area of operations, including coordinating the logistics and resupply of far-flung planetary bases. A number of logistics methods have been perfected by commercial and military experts, but these are not well understood in the context of space exploration. This paper describes a field expedition to a Mars analog site in the high Arctic, at which terrestrial logistics methods were tested in the context of (analog) planetary exploration. A comprehensive comparison is drawn between the logistics scenarios at HMP and a potential lunar or Mars base, in order to determine the extent of the analogy between them. It appears that the analogy is quite good in certain categories of supplies and shipment, but breaks down in others. When certain straightforward differences are accounted for, the data gathered from HMP can be used to validate and inform planetary base logistics models in support of future human lunar and Mars
\end{abstract} exploration.

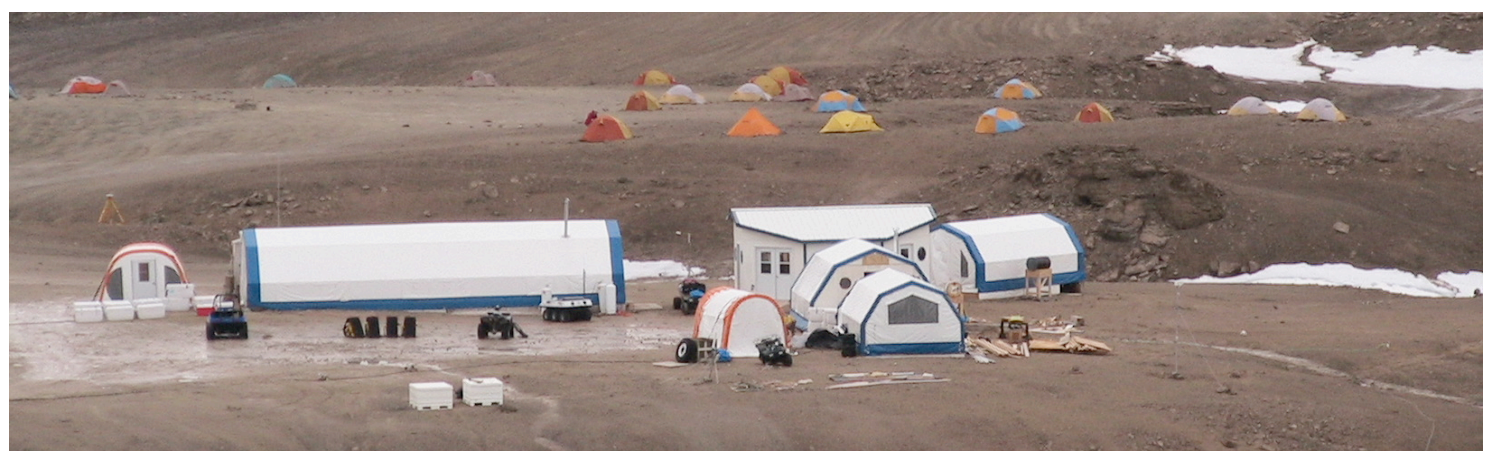

Figure 1. HMP Base Camp.

\section{Introduction}

Mission planners for future human space exploration enterprises (such as exploration of the Moon and Mars) face several major challenges in the area of operations. One of the least understood of these challenges is management of logistics and supply chains for remote planetary bases. As the world's space programs look ahead to human interplanetary exploration, the difficulties faced in past programs are compounded by the distance and complications of shipments of supplies, equipment, or spares to far-reaching destinations. Careful mission planning will be required to avoid shortages of crucial supplies and expensive, or even impossible, 'ondemand shipment' requirements. Sustainable space exploration operations are not possible without appropriate logistics management.

A vast number of scientific principles and techniques have been developed since World War II to improve the effectiveness and efficiency of terrestrial supply chains in the private and military sectors; this experience could be leveraged to improve space logistics operations. However, the potential benefits of this body of

\footnotetext{
* Graduate Research Assistant, AIAA Student Member, egralla@mit.edu.

† Graduate Research Assistant, AIAA Student Member, sshull@mit.edu.

* Graduate Research Assistant, AIAA Student Member, jaemyung@mit.edu.

$\S$ Graduate Research Assistant, AIAA Student Member, sidiqqi@mit.edu.

** Assistant Professor of Aeronautics \& Astronautics and Engineering Systems, AIAA Senior Member, deweck@mit.edu.
} 
knowledge are currently only poorly understood in the context of space exploration. In an effort to bridge the gap between terrestrial logistics expertise and space exploration, several terrestrial analogies for space logistics have been studied in detail. Some of the most relevant terrestrial supply chain networks are those that support operations in remote and challenging environments, such as the high Arctic. In this paper, we discuss the results of an in-depth study and field expedition at the CSA/NASA Haughton-Mars Project (HMP) research base in the high Arctic (75N 90W), which is being developed into the functional equivalent of a Mars base (see Fig. 1). The central goal of the study was to investigate the similarities between logistics for remote terrestrial sites and for planetary exploration, in order to support the development of new space logistics modeling principles and techniques.

\section{A. Space Logistics Past and Present}

In the past, the term 'space logistics' has been understood to mean the organizational effort behind assembling all the required supplies for a space mission - such as laptops, socks, and dinners - determining what is needed, and perhaps shipping supplies to the International Space Station (ISS). This is by no means a simple task, and those in charge preside over a tangle of complicated paperwork, various interweaving databases, and competing demands on a very small cargo allowance.

With the next generation of space exploration programs, this situation will become significantly more complex. With the advent of human exploration of the Moon and Mars, the bases will be farther away, shipments more expensive and difficult, and supply requirements more varied and difficult to predict. Shipping spare parts to the ISS after a critical failure is relatively easy compared to the complications of sending urgently needed spares to a base on Mars. With this added level of complexity, logistics planning must be an exact science to ensure that critical shortages do not occur.

Logistics encompasses a wide variety of activities in support of space operations, including (1) determining what is needed, (2) assembling supplies, (3) shipping supplies, and (4) tracking location and consumption of supplies on-base. It is critical that these activities be accomplished in a manner that is both affordable and sustainable. Logistics is the backbone of any space exploration mission, as it includes the transportation of all goods and supplies from Earth to a planetary or orbital destination. As a result, logistics must be accomplished in the most cost-efficient manner possible. While, as mentioned above, commercial and military organizations have successfully refined their logistics processes to minimize cost, the space community has not yet focused on this area. We aim to remedy this situation, by drawing lessons from the terrestrial logistics community to support new techniques in space logistics design and analysis.

We can learn the art of space logistics through detailed study of terrestrial logistics networks that bear a strong resemblance to space exploration. The techniques exploited to optimize supply chains to remote military bases, for example, can be adapted to remote bases on the Moon or Mars. In this paper, we focus on a detailed study of operations and logistics management at a remote scientific base in the Canadian Arctic, called the Haughton-Mars Project (HMP). Its scientific mission, remote location, and fragile supply chain suggest that it provides a strong analogy to space exploration. Through a thorough study of HMP's operations and supply chain, we can understand how terrestrial logistics is accomplished and apply the same techniques to space exploration.

\section{B. Research Goals and Overview}

The over-arching goal of this research project is to learn the art of space logistics, based on past experience in both space and terrestrial logistics. Lessons learned from past space logistics experience is covered in a separate publication ${ }^{1}$. Learning from terrestrial logistics - the focus of this paper - can be considered a two-step process:

1. Study Analogies: Understand how logistics is accomplished in terrestrial situations by studying analogous supply chains - military, commercial, and exploratory.

2. Adapt Techniques: Adapt the techniques used to manage terrestrial supply chains for use in planning and operating interplanetary supply chains.

This paper goes through those steps in detail for our study of the HMP supply chain. The specific goals of each step are outlined in the following paragraphs. 


\section{Study Analogies}

We first study the specialized logistics requirements that arise due to HMP's extremely remote location. Specifically, we seek to quantify the types of equipment and supplies present on base, and record their mass and volume. This provides a rough estimate of the cargo requirements of a planetary base. In addition, we study the operations scenario by recording the supply chain network and the flow of cargo and people through the network. We must then understand the extent of the analogy between HMP and a potential lunar or Mars base. The data gathered is therefore compared to estimations for lunar base cargo requirements, and the validity of the analogy can be estimated. Finally, for those areas in which the analogy holds true, we can validate Moon/Mars base logistics models against the data from HMP. In addition, we can understand which HMP operational techniques can be useful in the planetary exploration context.

\section{Adapt Techniques}

Second, we seek to validate the use of terrestrial techniques in the context of space exploration. We therefore test terrestrial logistics methods - garnered from study of HMP and other terrestrial supply chains - at the Haughton Mars Project. This research base provides a close operational analog to planetary exploration, so techniques that are effective in managing HMP logistics should prove useful in the interplanetary context. Specifically, we study the effectiveness of network modeling and optimization techniques ${ }^{2}$, and test a customized set of exploration supply classes in the field. The potential of radio-frequency identification (RFID) is explored in a separate paper ${ }^{3}$. With the data gathered from our study of HMP operations, we can also refine and (hopefully) validate current assumptions about operations at a planetary base, including the operational support (e.g. inventory tracking $\left.{ }^{4}\right)$ and cargo/equipment requirements.

The remainder of this paper explores each of these goals in detail. First, the research procedures are discussed in detail, including the equipment inventory and network modeling efforts. Second, the results of the field expedition are summarized, showing the distribution and amount of equipment types on base, and the personnel/cargo flows through the supply chain. These data are compared to the predictions of a parametric model for lunar bases, and the extent of the analogy between HMP and lunar bases is extrapolated. Finally, with an understanding of the similarities and differences between HMP and planetary bases, we discuss the effort at refining and validating current planetary base models against HMP data, and the adapting of terrestrial logistics techniques in the exploration context.

\section{Background}

The Haughton-Mars Project (HMP) provides an excellent opportunity to study a fairly close analog to a planetary research base. HMP allowed us to gain an in-depth understanding of how logistics is managed for a remote science camp, and also acted as a 'terrestrial laboratory' to test the application of commercial and military supply chain management techniques is an exploration context. Specifically, we wanted to understand how logistics is managed for remote base operations on Earth, and identify where terrestrial logistics analogies break down when applied to space exploration. Thus, one of our major goals was to quantify the extent of the analogy between HMP and potential lunar or Martian bases.

The following paragraphs provide an overview of the goals and operations of the HMP research base.

\section{A. The Haughton-Mars Project}

\section{HMP Location and Mission}

As mentioned above, HMP is a science research camp located in the high Arctic (75N 90W), on Devon Island in the Nunavut territory of Canada (see Fig. 2). HMP was established to study the Haughton impact site (see Fig. 3), whose geologic features and remote, desert setting provide a solid analog for potential research activities as a Martian base. Thus, while HMP supports a strong scientific mission (including geology, astrobiology, etc.), the base is also invested in developing new exploration techniques that can be applied to lunar and Mars exploration.

From the perspective of the space logistics study, HMP is an excellent place to study a terrestrial supply chain analogous to space exploration. The base has a similar mission to a planetary exploration base, so the camp should stock the same kinds of supplies one would find on a lunar or Martian base. In addition, the remoteness of the site requires a complex supply chain to keep the base in operation. Finally, the operations concept of the base parallels certain aspects of lunar and Martian surface missions. 


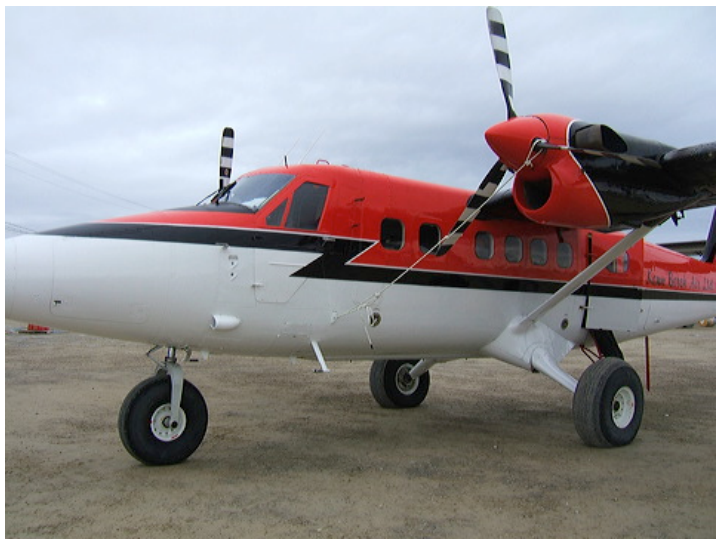

Figure 4. Twin Otter planes are the only reliable transportation to the HMP base.

\section{HMP Logistics.}

The HMP base can be accessed only from the small Inuit town of Resolute Bay (see Fig. 2). Twin Otter propeller planes are flown from the Resolute airport to a landing strip near the HMP research station (see Fig. 4). This is the only reliable form of transportation for people or goods into or out of HMP camp. In the past, air drops have been arranged, but this cannot be counted upon. It is only slightly simpler to access Resolute Bay. Northern airlines fly mid-size jets from large cities such as Ottawa to towns in the North, including Resolute. The only viable alternative is a slow-moving but inexpensive barge whose cargo must be loaded two years before it arrives in Resolute. This time scale makes it impractical for HMP.

In observing operations during the set-up of the HMP camp in the summer of 2005 , it became clear that logistics is organized in an ad-hoc fashion. The large quantity of cargo required to open the field season is sorted by participants at the Resolute Airport into loads for each of the five or six Twin Otter flights required to transport everything to Devon Island. The loads for each plane are sorted so that if no further flights are allowed that day, those on base can survive without the equipment left behind. Flight schedules are apt to change any minute due to the fickle weather, or the requirements of the other science teams dispersed around the Arctic during the summer field season.

Because of the remoteness of the HMP site, and the complex supply chain required to support its operations, the HMP research base can be supposed a good analogy for interplanetary supply chains; this supposition was tested during this study. Furthermore, because HMP's science and exploration mission parallels that of potential lunar or Martian bases, the site should provide a good analogy in terms of supplies, equipment, and infrastructure as well as logistics. Therefore, in order to understand terrestrial logistics management and to test logistics analysis techniques on a space exploration-like environment, we studied operations at the HMP research base in depth.

\section{Research Procedures}

Recall that the two major goals of the HMP expedition were to study the management of operations and logistics at the HMP base camp, and to test the application of terrestrial logistics techniques in an exploration context. In support of these objectives, we had two more specific goals:

1. Inventory and Classification: Quantify the types and amount of equipment and supplies on base.

2. Network Modeling: Record and model the operations scenario and people/cargo flows through the supply chain.

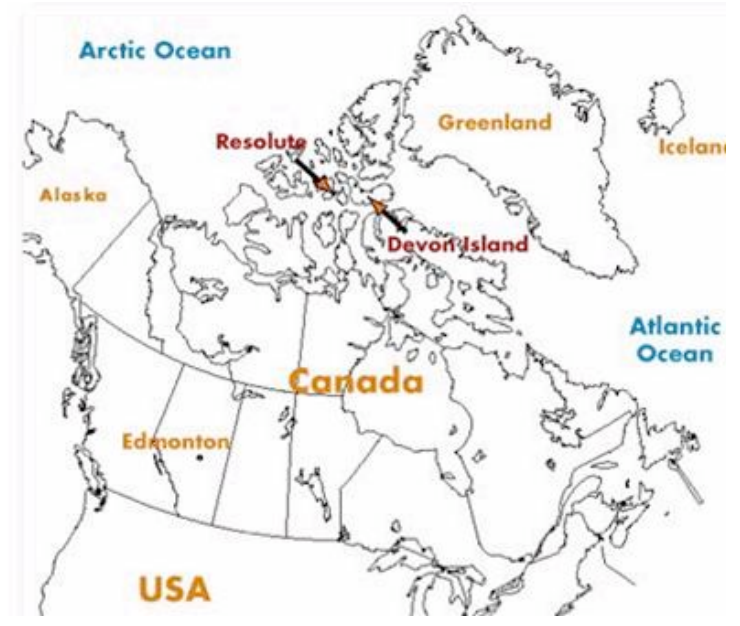

Figure 2. Map of Canada, showing the locations of Devon Island and Resolute Bay.

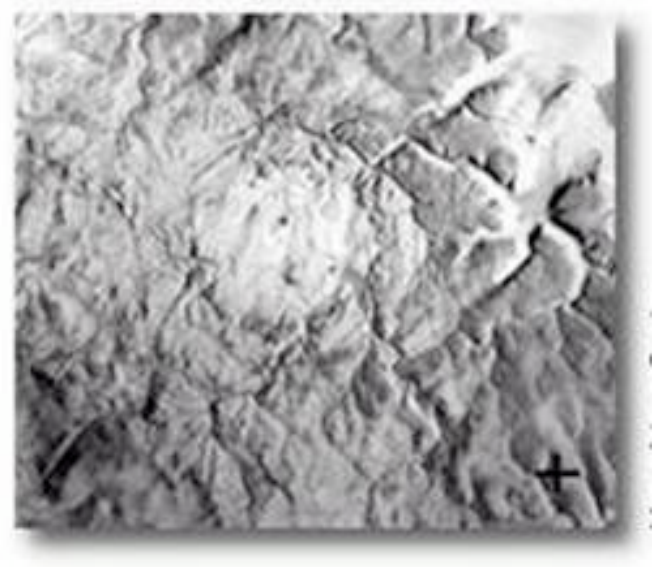

Figure 3. The Haughton impact site. 
In the following sections, we describe the procedures used to determine a classification scheme, perform a near-complete inventory of the base, and model the operations and supply chain network.

\section{A. Inventory and Classification}

\section{Classification of Supplies.}

In order to determine the types and amount of equipment on base, it was necessary to devise a consistent scheme for classifying various equipment items. This classification scheme had to be comprehensive, nonoverlapping, and allow us to compare the inventory numbers to models for each of these classes of supply. In addition, it is desirable that these classes of supply either stem from or map easily to a standard classification, such as that currently used by NASA.

Unfortunately, none of the standard supply classification schemes are suitable for exploration logistics. The classes of supply (COS) of several organizations proficient in remote logistics were examined, including NATO, the U.S. Military, and NASA. It was determined that none of these schemes had a uniform classification method for all items in the supply chain, and all had problematic deficiencies for exploration logistics. For example, NASA's CCART scheme does not contain categories for propellants, surface infrastructure (such as habitats), or surface exploration equipment.

A new function-based generic COS classification was therefore formulated that would serve the requirements of an interplanetary exploration supply chain. The COS were derived based on an object-process diagram, shown in Figure 5, which highlights the high-level functions (processes) of an exploration enterprise, and identifies their associated objects. For example, the human crew (object) performs the function of exploring/researching (process), which requires exploration and research equipment (object). The primary objects identified in this analysis are grouped to form the basis of a new functional classification scheme.

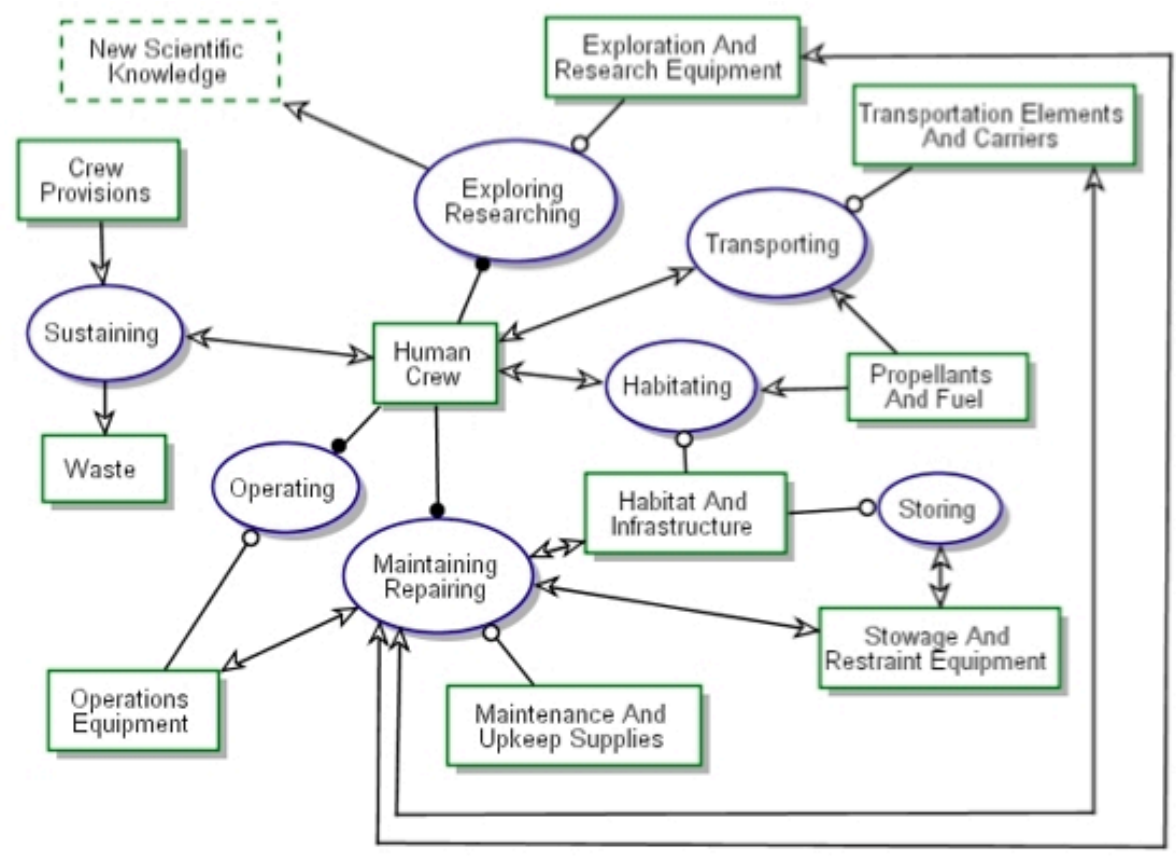

Figure 5. Object-Process diagram of a generic exploration system.

\footnotetext{
* Details on the analysis of NATO, US Military, and NASA (International Space Station) classes of supply are beyond the scope of this paper. Further information can be found in [5] Section 3.1 and Appendix B.
} 
The ten functional classes of supply are shown in Figure 6. Each of the ten classes has several sub-classes which further refine the categorization of the supply item. By assigning each item to a supply class and subclass, we allow a flexible multi-level supply class hierarchy which can give us great flexibility in dealing with supply classes at different levels of granularity.

At HMP, through the field inventory, we were able to validate this functional view of exploration supply classes, and ensure that everything found at base could be categorized sensibly and coherently. Physically recording the detailed inventory (described below) also guided the development of a total of 44 subclasses that are complete, non-overlapping, and reflective of the physical reality encountered at the research station. The complete list of classes and sub-classes of supply is given in Appendix A.

\section{Base Inventory.}

The development of the supply classes enabled a near-complete inventory of equipment and supplies at the HMP base camp. The inventory was intended to generate a model of base operations, which could be analyzed as an analog to a Moon or Mars base. Therefore, the field team attempted to inventory all equipment and supplies that were both accessible and relevant to the analog analysis.

An Excel-based inventory file was used to record all items examined, including relevant attributes such as weight, volume, description, location, and supply class. Based on field observations, we also added a 'percent full' attribute to enable tracking of fuel levels (e.g. empty, partially full). Almost every location at camp was inventoried, including the MIT work tent, bathroom tents, HMP office tent (including medical supplies), research tent, core module, mess tent, greenhouse, communications and computing storage tent, Humvee Rover, and outdoor areas. In each location all items used for base support or science purposes were inventoried. We did not systematically inventory personal items that were kept in any locations, nor the contents of personal sleeping tents (to avoid invasion of privacy). These items were later estimated based on the personal inventories of MIT team members.

Over 2300 items were inventoried by the 2005 expedition team, encompassing nearly all relevant locations. These data provide an idea of the aggregate mass and volume of various types of equipment in residence at HMP. These results also enable comparisons between HMP equipment totals and planetary base cargo mass and volume estimates from parametric models.

\section{B. Network Modeling}

The final major research thrust was to record the operational scenario and supply chain network flow for the HMP base. The supply chain network itself is relatively straightforward, and is shown in Figure 7. Teams and equipment depart various locations around the world, fly to Resolute Bay (node 3), and from there fly to the HMP base on Devon Island (node 5). Note that all people and cargo flow through the Resolute-Devon link. In addition, this is the only link over which HMP has direct control (airline prices and schedules are generally 
fixed). Therefore, in the remainder of this analysis, we focus on the Resolute-Devon link in our attempts at studying and optimizing the HMP cargo flow.

The flow through this network was tracked by recording the manifests of each Twin Otter flight along the Resolute-Devon arc, including both people and cargo. In many cases these data were difficult to obtain, due to the rather ad-hoc nature of cargo loading and unloading. Inclement environmental conditions and extreme time pressure often foil attempts at documenting cargo manifests, and the load is simply weighed in aggregate to ensure it remains below the plane's cargo capacity. However, by observing each flight's loading or unloading, a $\log$ of all flight manifests (a best estimate) was created. This allows the estimation of cargo and personnel flows through the supply chain for the entire field season.

\section{Results \& Analysis}

With the data gathered as described in Section III above, we have a rich base for understanding the operations and logistics scenario at the Haughton-Mars Project research base. We first study the results of the inventory and network modeling efforts, then compare these data to parametric planetary base models to analyze the extent of the analogy to HMP.

\section{A. Inventory and Network Modeling}

The inventory and network modeling studies give us an in-depth view of the operations of the HMP research base. We can study these data to garner lessons that address our original goal: learn the 'art' of space logistics from terrestrial analogs. This quantitative analysis of HMP logistics will be used to inform operational planning for planetary base missions.

\section{Inventory}

In order to determine the distribution of types of supplies at HMP, the inventory is analyzed in terms of classes of supply. Figure 8 shows number of items in each subclass of supply that resided at HMP during some point in the 2005 field season. Only the largest subclasses of supply are shown. Nearly one fourth of the entire inventory at the research station was dedicated to food and related equipment $(\operatorname{COS} 2.2,23 \%)$. The second quarter was comprised of communications equipment (COS 3.5, 15\%) and science instruments (COS 6.1, 11\%), followed by health-related equipment $(\operatorname{COS} 3.3,9 \%)$. This last supply class may be surprising, but HMP

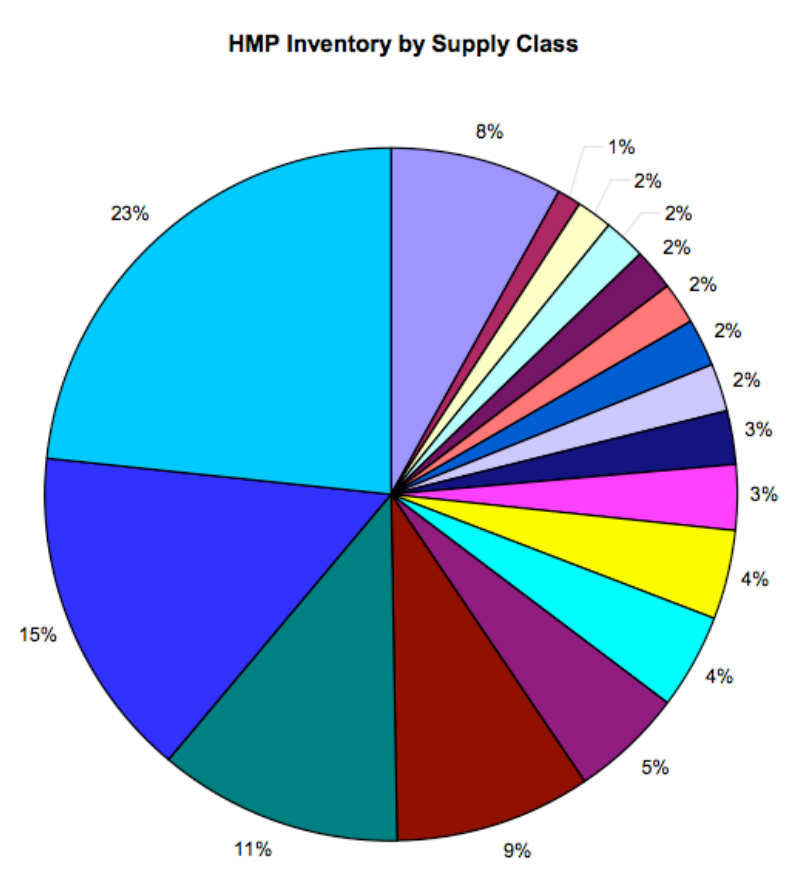

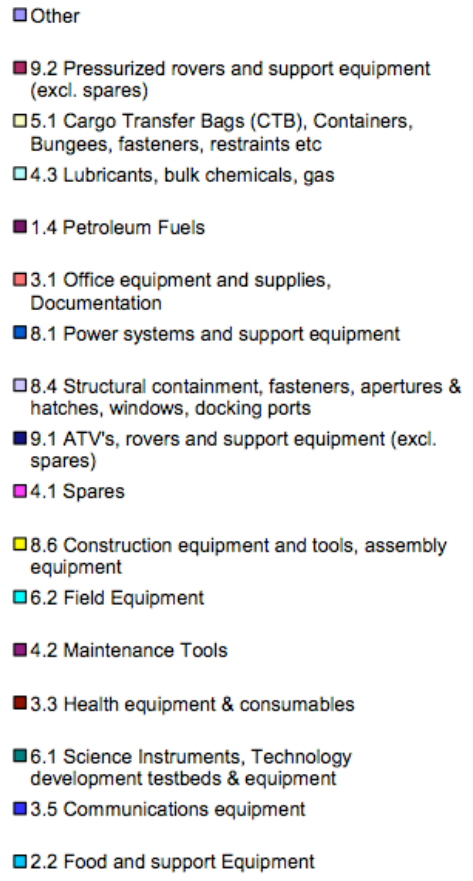

$\square 2.2$ Food and support Equipment

Figure 8. HMP inventory by supply sub-class (counterclockwise). By number of items. 

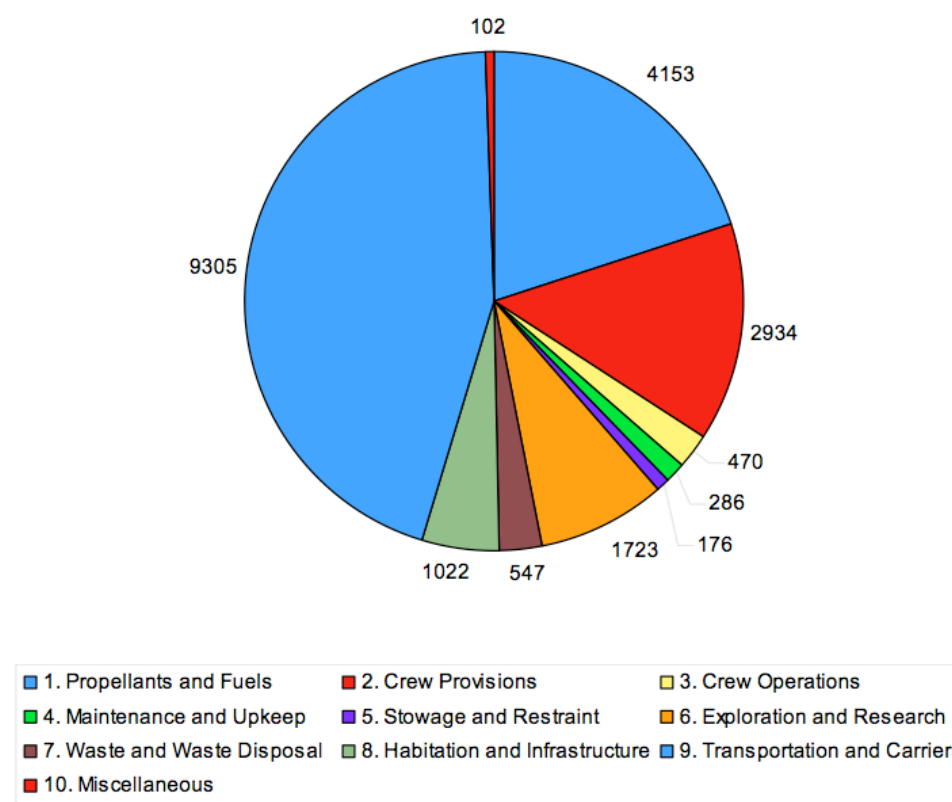

Figure 9. HMP mass breakdown by class of supply [kg]. (Clockwise)

maintains a high level of medical capability, both through personnel (certified flight surgeons and expedition doctors) as well as a variety of expeditionary medical supplies and sophisticated equipment (e.g. Canadian Space Agency telemedicine kit).

The inventory was then analyzed in terms of mass rather than number of supply items. The total mass recorded at HMP was $20,717 \mathrm{~kg}(\sim 46,000 \mathrm{lbs})$. Figure 9 shows the mass breakdown among the ten classes of supply. We note that transportation items (COS 9, 46\%) dominate the total mass of supply items at HMP. This is primarily due to the presence of 13 All-Terrain Vehicles (ATVs) and one Humvee: each ATV weighs between $227-325 \mathrm{~kg}$ and the Humvee alone has a curb weight of at least $4700 \mathrm{~kg}$. This COS is followed by the aggregate mass of the various fuels and propellants that are stored at the HMP research station (COS 1,20\%), crew provisions $(\operatorname{COS} 2,14 \%)$ and exploration and research equipment $(\operatorname{COS} 6,8 \%)$.

It is clear that the classes containing the largest number of items are not always those with the largest mass. These metrics drive two different requirements on a planetary base: high-mass items are difficult and expensive to transport to the base, and drive a significant portion of the cost of logistics. On the other hand, low-mass items that are required in large numbers are relatively easy to ship and to replace, but often difficult to track. The reason that so many individual food "items" (cans, boxes, jars) reside at HMP is that food has not historically been tracked from season to season, so each summer a completely new shipment of food arrives, and the leftovers remain unused, building up a large (useless) inventory. Furthermore, while it is perhaps allowable to lose track of individual food items, the tracking capability is essential for critical items like medical supplies, which can expire over time.

The HMP inventory therefore highlights the areas in which logistics improvements should be researched. Reducing the mass of the heaviest classes of items - surface transport vehicles and their propellants - could significantly reduce in-space transportation costs. In addition, implementing an effective tracking system for supplies like food and medical equipment could reduce the build-up of large excess stockpiles and alleviate some of the danger of critical shortages. These are important conclusions for space logistics.

\section{Network Modeling.}

The network modeling effort can be summarized by showing the calculated flow of cargo and people through the HMP supply chain network. Figure 10 shows the cumulative cargo flow into Devon Island (from Resolute) for the 2005 field season.. The total cumulative network flow was $22,750 \mathrm{~kg}$, corresponding to an average flight cargo capacity utilization of about $73 \%$ (inbound). The total flow out of HMP was only 12,430 kg, an average capacity utilization of $40 \%$ (outbound). Clearly, more cargo is brought into HMP than is removed. Much of this mass is construction equipment and other permanent infrastructure, but significant equipment 
build-up does occur. Personnel movement accounts for one third of the inbound cargo flow, while $61 \%$ of outbound flow is attributed to people.

This analysis highlights areas in which operational improvements could be made to optimize base logistics. As mentioned earlier, the Resolute-Devon transportation link has the largest potential for improvement. We have just concluded that the inbound and especially outbound flight capacities are under-utilized. A simple form of optimization finds the minimum number of flights required to transport the total incoming mass. This "ideal" flight schedule could save up to $33 \%$ in terms of number of flights and therefore transport costs. The key point here is that applying a terrestrial logistics technique - network modeling and optimization - in an exploration context does indeed highlight areas which can be improved in the operational scenario.

\section{B. Comparison to Lunar Base}

The final step is to take the entire set of data gathered during the field expedition, including the inventory, classification, and network modeling results, and draw comparisons to expectations for lunar planetary bases. We begin by describing the assumptions behind the parametric models for lunar bases (both short and long missions), then compare the model outputs to the inventory data collected at HMP. Comparisons are first broken down by class of supply. For the more interesting supply classes, we also provide a comparison by subclass. The conclusions from this comparison indicate the extent of the analogy between HMP and lunar or planetary bases.

\section{Modeling Assumptions.}

Surface 'demand' models were developed for a NASA CE\&R study at MIT $^{6}$. The models estimate the required supplies (by mass) for surface missions on the Moon and Mars. Separate models incorporate assumptions appropriate to 'short' lunar missions (approximately 10 days on the surface), 'long' lunar missions (approximately 180 days), and Mars missions (600 days, not considered here). The basic assumptions and numbers for the long and short lunar models are based on relationships in $\mathrm{HSMAD}^{7}$, which are in turn based partly on data from experience with manned spaceflight programs. Details for our models can be found in [6] Appendix D.2a, along with references to specific sections of HSMAD where appropriate. The long and short mission models both take as input the number of crew and the mission duration, but they make different assumptions about the science equipment available, number of EVAs, etc. For example, the long mission assumes a larger allowance of clothing and office equipment per crew-day, and calls for a pressurized surface rover. For the HMP work, the CE\&R lunar models were adapted to generate estimates of required equipment within the ten supply classes. The comparisons below are based on the short and long lunar surface demand models.

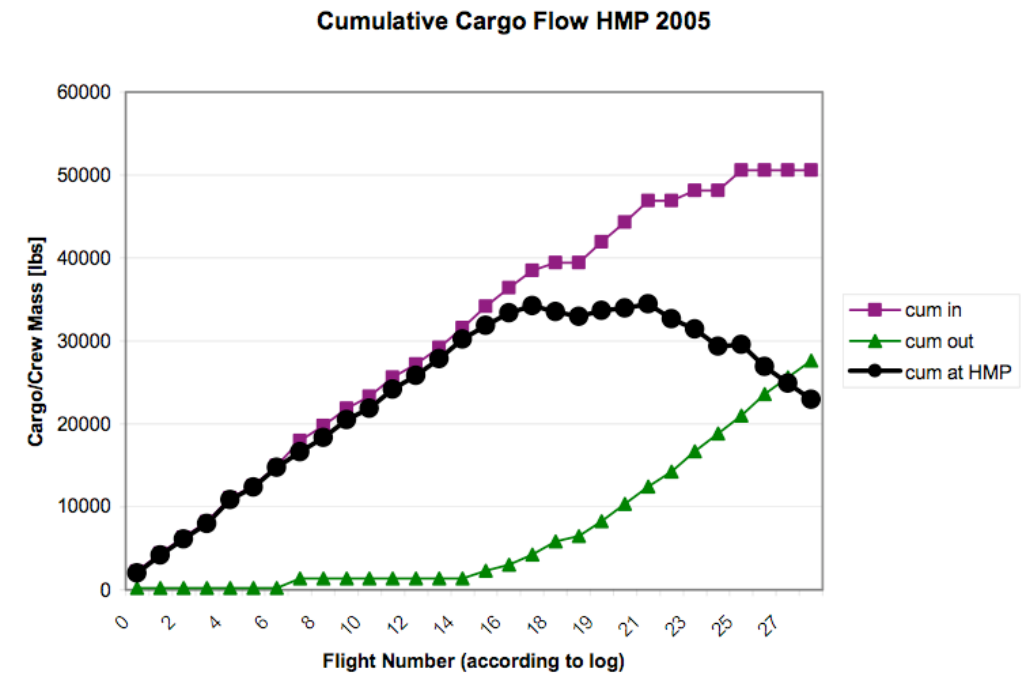

Figure 10. Cumulative mass flow in and out of HMP for the 2005 field season. 


\section{Comparison by Supply Class (Full Data Set)}

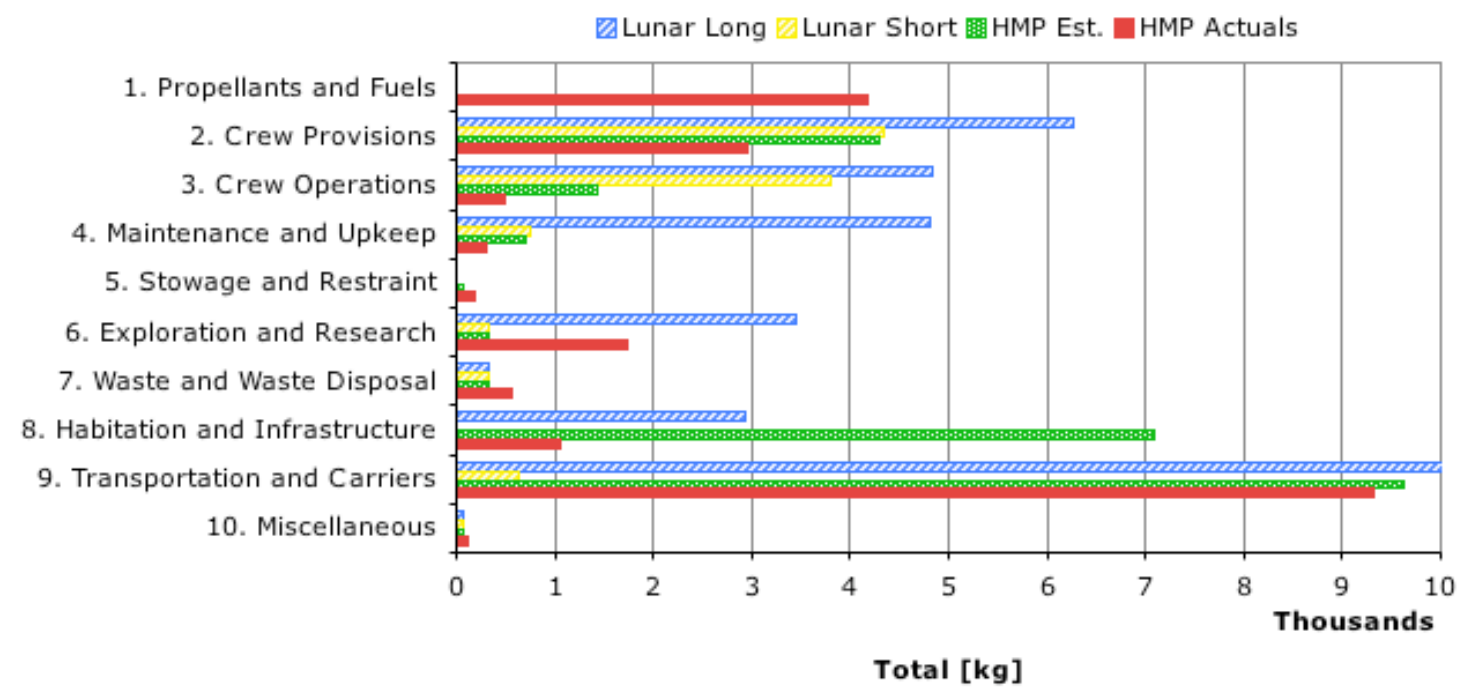

Figure 11. Mass comparison between HMP actual data and estimates based on a parametric exploration base demand model. The models all assume 19 crew members for 36 days (to line up with the 2005 HMP field season.

For the purposes of comparing the HMP inventory data to a 'best estimate', a third model was created by customizing the short and long lunar demand models. Based on a priori information about the mission and capabilities of the HMP research base, a model was developed utilizing the same basic relationships as the lunar demand models, but incorporating other HMP-specific factors. For the most part, the modeling assumptions for each supply sub-class are the same as those in the lunar short and long mission models. Wherever the lunar short and long models differ, a choice was made between them based on the supply sub-class. For personal items and certain research-related equipment, the short mission model is more appropriate, because each person at HMP is generally on a short-style mission (i.e. in residence for $\sim 12$ days rather than 180 days). However, for infrastructure and some permanent science equipment, HMP bears greater similarity to the long missions (which build up permanent infrastructure) rather than the short sortie-style missions. In some cases neither the short nor long lunar missions was an appropriate model. Estimates for specialized HMP equipment (such as ATVs or generators) were added to the model in that case.

Other HMP-specific estimates have also been included: the number of EVA suits (to be tested at HMP), tents (assuming a mass of $1000 \mathrm{~kg}$ for each of the seven tents), and vehicles, for example. Thus, the HMP estimate is intended to represent the best pre-expedition surface demand model that could be created for HMP given the current level of knowledge and modeling ability for human interplanetary bases.

In the following sections, each of these three demand models is compared to the results of the actual HMP inventory in order to assess both the extent of the analogy between HMP and lunar bases, and the accuracy of our current planetary base modeling methods. To make the numbers comparable, each model was given the inputs of 19 crew (average for HMP this season) and 36 days (the length of the field season).

\section{Comparison by Class of Supply.}

Figure 11 shows a comparison by class of supply between HMP actual inventory data (red bars), pre-HMP expedition estimates (green bars), and the lunar short and long models (yellow and blue bars, respectively). Several differences are apparent: the most obvious such difference is COS 1, 'Propellants and Fuels'. Our HMP estimate for propellants and fuels was zero based upon the fact that these items were accounted for differently in our previous CE\&R work $^{6}$ and are mainly associated directly with in-space transportation vehicles (e.g. the $\mathrm{CEV}$ ). Additional work is required to distinguish between fuels and propellants used for transportation to and from the base, and fuels used while on base (including for surface mobility). A large difference can also be noted in the maintenance and upkeep (COS 4), exploration and research (COS 6) and habitation and infrastructure (COS 8) classes. These differences are explained in the detailed breakdowns by subclass, below. 


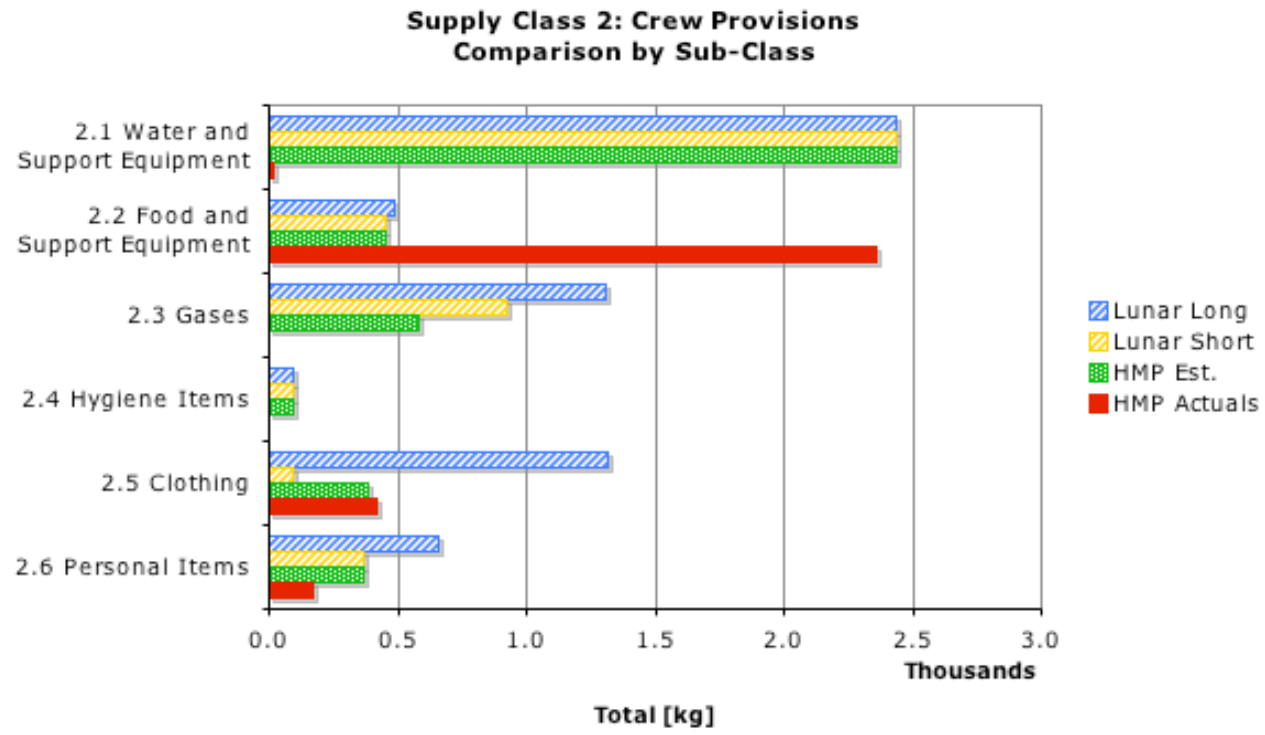

Figure 12. COS 2 Comparisons by Sub-Class.

COS 2: Crew Provisions. From Figure 11, it appears that the HMP estimate for class of supply 2, crew provisions, is about $1300 \mathrm{~kg}$ larger than the HMP actual inventory. When COS 2 is broken down into its subclasses (Figure 12) the sources of this difference become apparent.

We see from COS 2.1 that almost no water is imported to HMP in actuality because of local resource usage, whereas the models predict that $2428 \mathrm{~kg}$ of water would have to be shipped in to support the field season. On the flipside, the comparison for $\operatorname{COS} 2.2$, food and support equipment, shows the HMP actual figures outweighing the HMP and lunar estimates by nearly $1900 \mathrm{~kg}$. HMP has stockpiled several seasons' worth of food, as well as a wide variety of kitchen equipment. Thus, the HMP food actuals are a significant overestimate of actual integrated HMP food consumption rates. Another contributor to the difference is that spaceflight estimates are based on the use of dehydrated food ( $0.64 \mathrm{~kg}$ per person per day), which is much lighter.

$\operatorname{COS} 2.3$ comprises gases that must be provided for crew consumption (oxygen, nitrogen, buffer gas). Obviously, gases are used directly from the environment at HMP and the pre-HMP estimate should have already reflected this. Personal hygiene items (COS 2.4) were certainly present at HMP but were not inventoried, since they were kept in the individual sleeping tents. The clothing (COS 2.5) estimates for lunar missions are based on clothing allowances which take into account the lack of a washing machine. The HMP estimate falls somewhere between, due to slightly longer stays and requirements for heavy outdoor clothing. Outdoor clothing actuals for HMP were estimated here based on the luggage allowance for the airline flight to Resolute; these actuals line up surprisingly accurately with the estimate. Personal items (COS 2.6) which resided in personal tents were not inventoried for privacy reasons, so the actuals fall behind the estimate.

For crew provisions (COS 2) in general, we conclude that local availability of water and oxygen at HMP are the main contributors that make HMP non-analogous to the lunar demand models. We could change the lunar models to include the use of ISRU, but this would also require adding an ISRU plant and associated power system, which would be needed on the Moon and Mars, but not at HMP. The food comparison shows that HMP is also not analogous to space exploration, mainly because it does not use dehydrated foods and has created a large cache of (heavy) canned food. The other items (hygiene, clothing, and personal) are quite analogous.

COS 3: Crew Operations. In Figure 11 the estimate for HMP Crew Operations Equipment (COS 3) exceeded the actual recorded inventory at HMP by about 1 metric ton. From Figure 13 we see that this difference is primarily driven by COS 3.2, EVA Equipment and Consumables. The HMP estimate takes into account that EVA suits are not required for operations on Earth (as they are on the Moon) but assumes that five EVA suits (with associated consumables) would be tested at HMP. In fact, the single Hamilton Sundstrand test suit brought to HMP in 2005 turned out to be much lighter. Actual and predicted office equipment (COS 3.1) was below 500 $\mathrm{kg}$. 


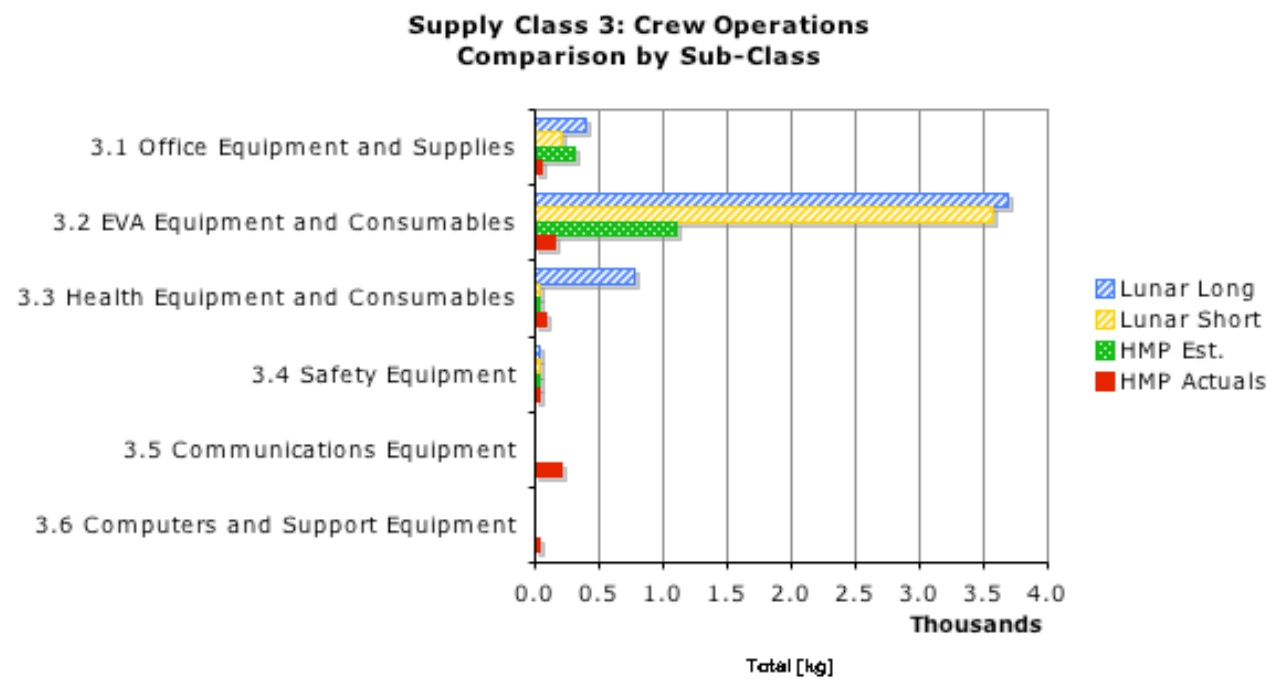

Figure 13. COS 3 Comparisons by Sub-Class.

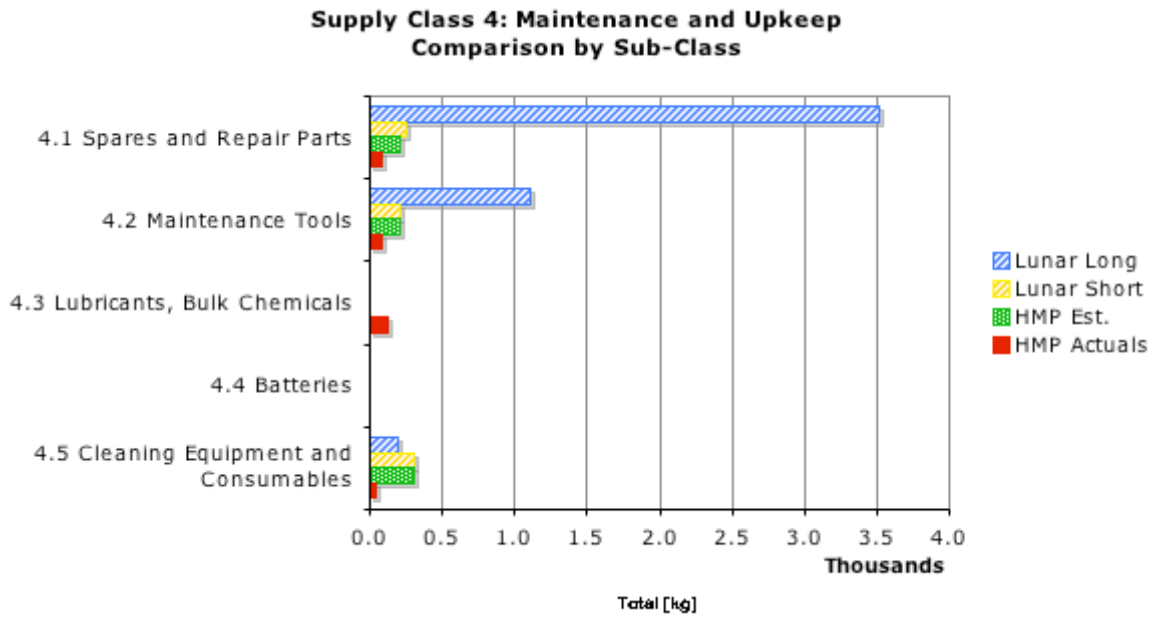

Figure 14. COS 4 Comparisons by Sub-Class.

Health (COS 3.3) and Safety (COS 3.4) equipment is essential, but it is not very heavy or bulky. The estimates and actuals do not seem unreasonable, given that HMP medical supplies are quite basic in nature.

The lack of mass data around communications equipment (COS 3.5) and computers and support equipment (COS 3.6) was somewhat puzzling at first. Clearly, HMP has a suite of sophisticated communications gear (Cband satellite transceiver, parabolic antenna, on-site wireless network,...) but much of this type of equipment would be fully integrated into vehicles, habitats, and EVA suits in a lunar base, so it was not predicted in those subclasses for a lunar mission. We only accounted for this type of equipment under COS 4.1 (spares), rather than counting communications and computers as separate supply items. Clearly, the inventory for this supply class shows that the relationship between items already integrated in various vehicles and truly "separate" supply items must be better understood in the future.

COS 4: Maintenance and Upkeep. From Figure 11 it appears that $\operatorname{COS} 4$, maintenance and upkeep, was slightly overestimated by the parametric HMP base exploration logistics model. The mass breakdown by subclass (Figure 14) shows the differences.

From Figure 14, we observe that the pre-HMP estimate for COS 4.1, spares and repair parts, turned out to be double what the actual recorded masses were. The two reasons for this difference are that (1) much of the maintenance at HMP depends on scavenging parts from in-use spares (taking parts from another ATV until a new one can be ordered), and (2) HMP employs the "order as needed" strategy, enabled by the Twin Otter flights 


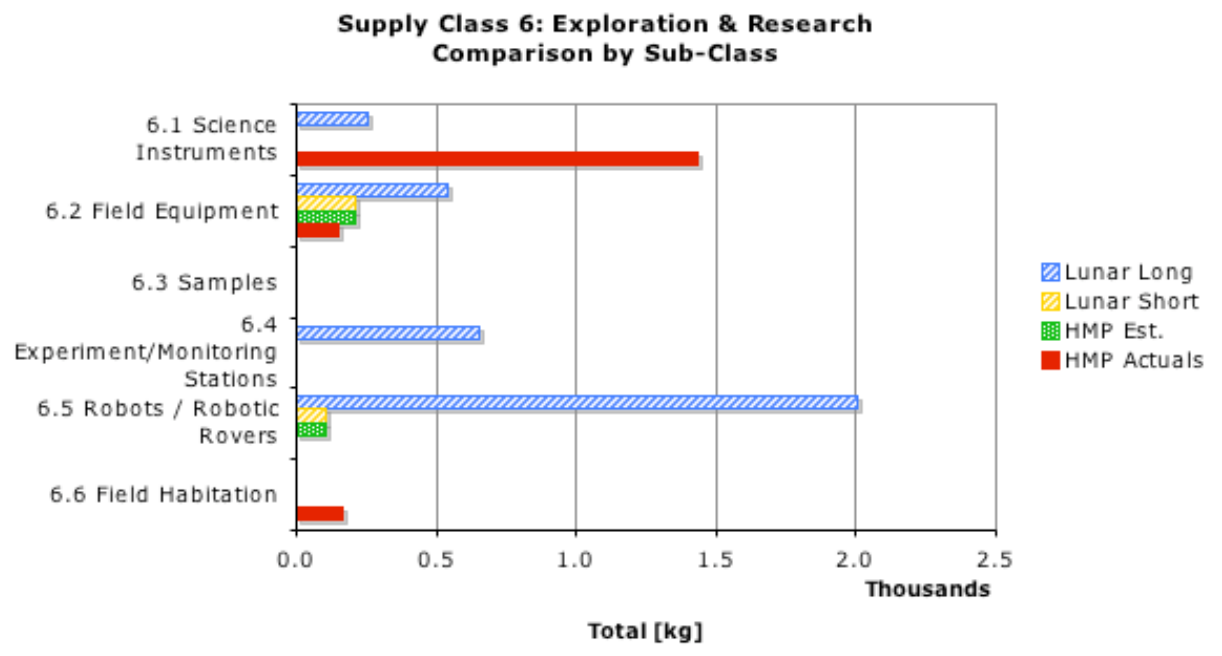

Figure 15. COS 6 Comparisons by Sub-Class.

to camp about twice a week. Essential spares can be acquired within a week of ordering. Clearly, such a strategy would not be possible for lunar missions and that is why significant amounts of spares would have to be taken along or pre-positioned (see large blue bars in Fig. 14).

Maintenance Tools (COS 4.2) exist at HMP, but they are generally light-weight and multipurpose, unlike the heavier and more specialized equipment that is typically used in manned spaceflight operations. Lubricants and chemicals (COS 4.3) and cleaning equipment and consumables (COS 4.5) turned out to be rather insignificant, both in the model and in actuality.

COS 6: Exploration and Research. This category of supply items is probably one of the most difficult to predict, as it depends very strongly on the particular scientific and technological objectives of an exploration mission. The HMP actuals show that quite a lot of mass on base is devoted to science instruments, far more even than the long lunar mission model predicts. One of the reasons for this is that at HMP, we classified a large number of items as scientific instruments (and technology testbeds) that also serve "household functions" (communications, expeditionary medicine) and therefore could also have been classified under COS 3 . In addition, a major contributor to the HMP science instruments mass total was the $\sim 1400 \mathrm{~kg}$ of equipment in the greenhouse, which was not present in the long lunar mission model.

The field equipment inventory actuals line up well with our pre-HMP estimate and short lunar missions. We found that the field equipment used at HMP is in some cases an exact analog to what would be used for an equivalent Moon or Mars mission.

We did not take inventory of samples (COS 6.3) taken from the Haughton Crater, but know that such samples were obtained. Sample mass, however, is not as important to the logistics problem because samples are not transported to the base. The HMP actuals show zero mass for robots and robotic rovers, while we predicted a small mass consistent with the short lunar mission. In the past, such robots have been tested and utilized (e.g.

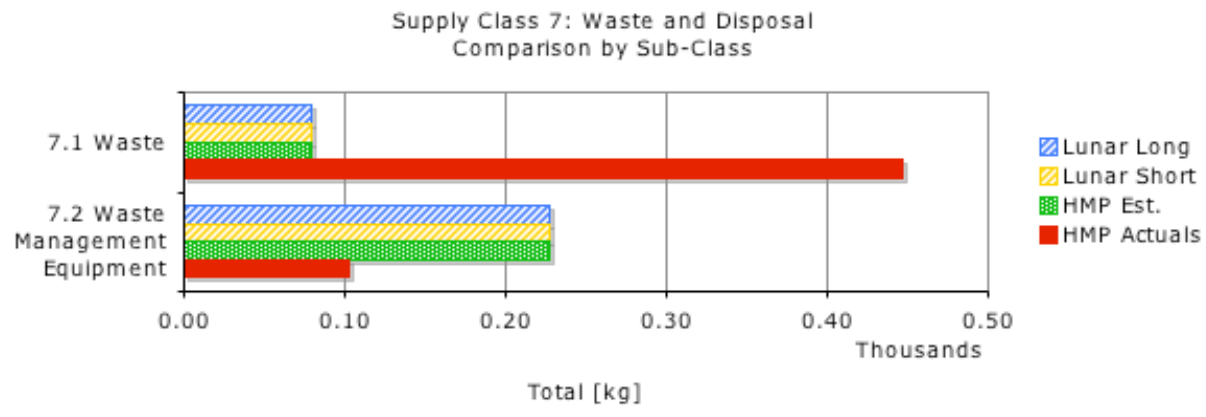

Figure 16. COS 7 Comparisons by Sub-Class. 
CMU's Hyperion rover). Field habitation (COS 6.6) at HMP consists mainly of tents/bivouacs for overnight stays away from base camp.

$\operatorname{COS} 7$ : Waste and Waste Management. This category showed an excellent correlation in Figure 11 between HMP and lunar/pre-HMP predictions in terms of mass $(303 \mathrm{~kg}$ vs. $547 \mathrm{~kg})$. This correspondence at the supply class level, however, masks some information that can be obtained by going to the sub-class level (Figure 16). The HMP actuals show a much larger mass devoted to waste, and a much lower mass devoted to waste management equipment, than predicted. This discrepancy is largely due to the difference in technological sophistication: the HMP system is quite simple, and depends largely on the use of trash bags for collection and storage of waste of all types (including human). On the other hand, a waste collection system for Moon/Mars bases would probably incorporate sophisticated equipment for water reclamation from urine, and attempt to minimize the use of disposable elements such as trash bags. Therefore, HMP shows a greater build-up of stored waste and a lesser focus on waste management equipment.

COS 8: Habitation and Infrastructure. From Figure 11, it can be observed that the actual HMP masses for $\operatorname{COS} 8$, habitation and infrastructure, are significantly under the estimates. This difference is entirely due to the differences in masses in COS 8.4, the structural containment. As noted earlier, we did not (yet) inventory the mass of the erected structures at HMP and they are therefore not included in the actual numbers. The MIT team determined that the core structure was not analogous to that which may be found at a lunar/Mars base (due to the extensive use of wood). The greenhouse structure was excluded for similar reasons. We expect that the HMP actuals will be heavier than the estimates largely because of the heavy materials used to construct the work tents. Power systems, heaters, and ventilation systems are relatively insignificant, and ISRU plants were not present at HMP. Construction equipment shows up in HMP actuals but not in the estimate because on a lunar base construction would be largely unnecessary (i.e. pre-constructed habitats).

COS 9: Transportation and Carriers. By far the largest category, in terms of mass, is represented by transportation vehicles on base. Here the correlations between HMP, our pre-HMP estimate and the long lunar mission is excellent, between 9-10 metric tons. Fig. 18 shows the mass breakdown for this category.

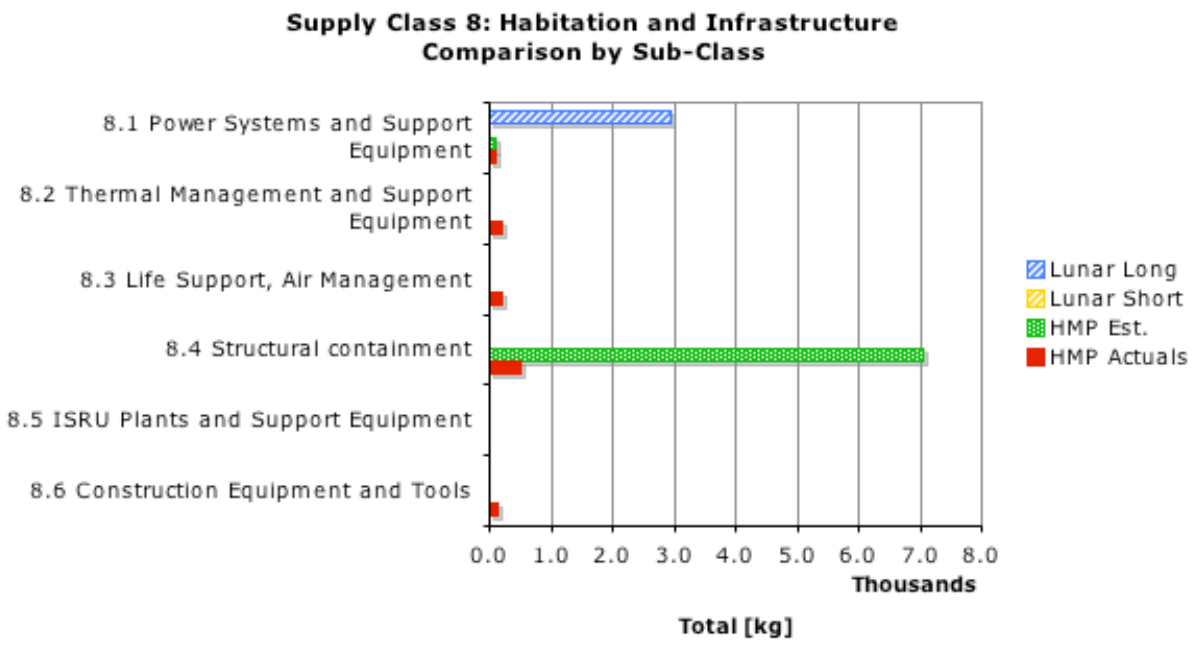

Figure 17. COS 8 Comparisons by Sub-Class. 


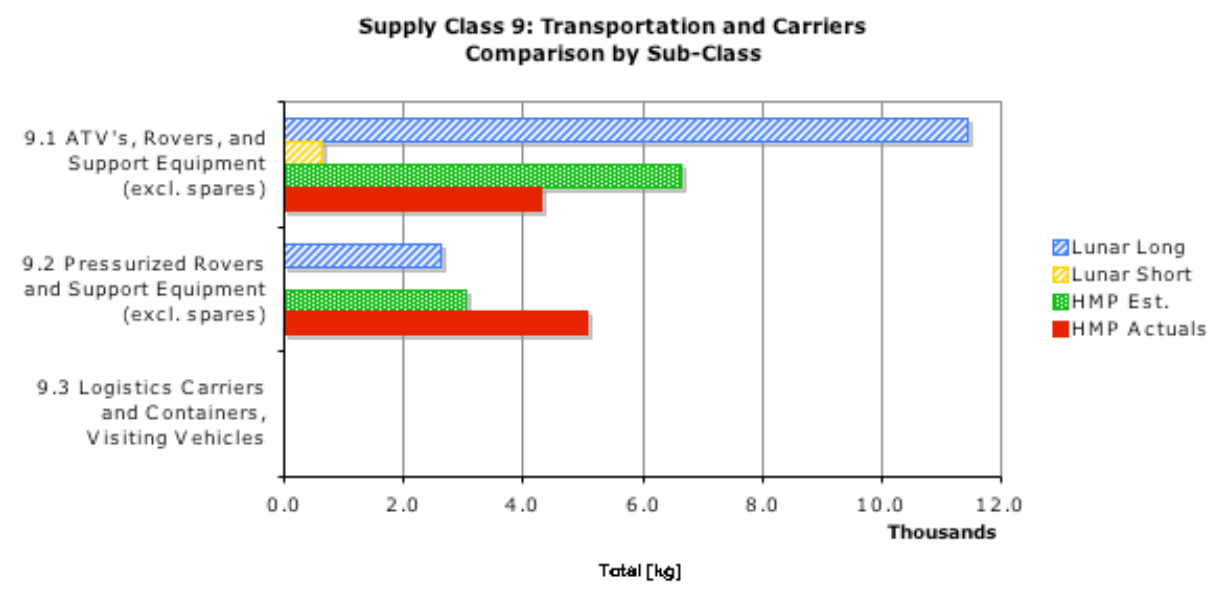

Figure 18. COS 9 Comparisons by Sub-Class.

COS 9.1 captures the ATVs and associated support equipment, excluding spares; the total mass of ATVs on base was slightly less than expected, but still significantly below that which would be required at a long lunar mission. The estimated mass of the MARS-1 Humvee is $4700 \mathrm{~kg}$ (Hummer catalogue value), which is more than the mass of its lunar analog, the long mission pressurized rover. Still, the actuals line up well with the estimates in this category, suggesting that our models for surface mobility are relatively valid.

Summary. In totality our actual HMP inventory yielded a mass of $20,717 \mathrm{~kg}$, versus $23,740 \mathrm{~kg}$ for the preHMP estimate. This is a $15 \%$ difference and is suggestive of the level of precision of our current planetary logistics requirements modeling capabilities. However, this conclusion might be somewhat premature as some items have not yet been included in the HMP inventory (e.g. tents) and we suspect that the actual inventory mass at HMP is closer to 25-30 metric tons. Still, with short term lunar mission requirements (Apollo-style) estimated at $10,082 \mathrm{~kg}$ and long lunar mission requirements estimated at $36,529 \mathrm{~kg}$ we see that HMP falls right in the middle between the two. Some aspects of HMP (short individual stays of 12 days on average) are more like the short-term Apollo-style missions, while others (permanent research infrastructure) are more like the proposed 180 day lunar missions.

\section{Conclusions}

As a result of the HMP field study, a near-complete inventory of the HMP base (nearly 2,300 items) was completed; the logistics network of flights and other supply channels was modeled; and the scientific mission of the base was studied. These three factors drive the logistics scenario for any remote base, and can therefore be compared to lunar and Mars bases in order to understand the extent of the analogy between remote terrestrial and interplanetary bases.

\section{A. Analogies between HMP and Lunar Base Logistics}

This section addresses the extent of the analogy between HMP and Martian/lunar exploration bases in terms of logistics. HMP was set up as a 'Mars analog base' in terms of science rather than logistics. HMP logistics is accomplished in whatever manner is easiest and/or least expensive. However, due to its remote location, the limited avenues of access to the base, and the similar demands on base infrastructure and supplies (supporting scientific researchers), the analogy between HMP and Martian/lunar base logistics is entirely reasonable.

We conclude that the analogy is more accurate in some areas than in others. For example, several obvious differences became apparent:

- HMP uses in-situ water resources. Water is imported from snow-melt feeding into the Lowell Canal nearby, and therefore it is unnecessary to bring in large quantities of water.

- HMP has more frequent flights (resupply) than a Moon or Mars outpost. Twin Otter airplanes can be flown in from Resolute Bay any time they are available (not all the time, but generally at least a few times per week). As a result, crucial consumables or unexpected equipment requirements can be shipped to HMP on relatively short notice, whereas the same is impossible on Mars/Moon. 


\begin{tabular}{|l|l|}
\hline Analogous COS w/Lunar or Mars & Not Analogous COS w/Lunar or Mars \\
\hline 2.4-2.6 Hygiene Items, Personal Items & 1.1-1.4 Types of Fuel used \\
\hline 3.1 Office Equipment & 2.2 Food and Support Equipment \\
\hline 3.3 Health Equipment, Telemedicine kit & 3.2 EVA Equipment \& Consumables* \\
\hline 3.5 Communications gear (C-band sat xpdr) & 4.1-2 Spares and Maintenance Tools \\
\hline 6.2 Field Research Equipment (cameras, ...) & 7.2 Waste Management System \\
\hline 6.3 Rock samples & 8.2-8.4 ECLSS, Thermal, Habitation \\
\hline 8.1 Photovoltaic power system (Greenhouse) & \\
\hline 9.1 ATVs (except for combustion engine) & \\
\hline 9.2 Pressurized Rover & \\
\hline 10.1 Public Affairs items (flags etc..) & \\
\hline With the exception of EVA suits brought in for testing, these, however, can also be classified under COS 6.1 \\
\hline
\end{tabular}

Table 1. Qualitative list of analogous and non-analogous supply classes at HMP.

- HMP is relatively 'luxurious' in cooking/eating arrangements. This is one area where HMP almost certainly differs from Mars/Moon. Because of the relative ease of shipments, standard food (e.g. fresh produce) can be flown in. Martian/lunar bases would almost certainly depend on dehydrated foods like those used on ISS today. The shipment masses would therefore be quite different for HMP, where heavy items such as canned food are shipped in.

- Criticality of Stowage Space. The availability and management of stowage space has turned out to be one of the most critical aspects of International Space Station operations. At HMP, on the other hand, there is an abundance of stowage space. This is primarily driven by the absence of a requirement for pressurized stowage at HMP; much cargo is stored outdoors, especially during the field season.

Despite these differences, we find that the remaining classes of supply provide a stronger analogy between HMP and Lunar/Mars logistics. These areas are summarized in Table 1. In addition, there are still lessons to be learned even in those areas that are recognizably different between HMP and Lunar or Mars bases.

\section{B. Analogy Conclusions}

Based on the analysis described in this paper, it is clear that our pre-expedition estimate of HMP cargo was relatively accurate, once known differences have been taken into account. The initial estimate predicts HMP stock levels within an order of magnitude for most supply classes. The total estimated mass of the HMP RS and its contents was around 23.7 metric tons, including a 7 metric ton allowance for structural containment, whereas the actual recorded inventory estimated around 20.7 metric tons.

This is a significant achievement, since modeling planetary bases is to-date largely a matter of educated speculation. No planetary bases have been built, and the best real data in existence is from the days of Apollo (with outdated technology and a different mission format) or by analogy with ISS which is a large micro-gravity facility. The data gathered at HMP allows surface expedition modeling relationships to be tested and verified against another set of real data on remote bases (with acknowledged differences between Earth-based and planetary setups). The lack of significant unexplainable differences between the lunar/Mars models and the HMP data suggests that the models are acceptable predictors of planetary base surface mission requirements, but that substantial refinement and additional parametric modeling will be required in the future.

Table 1 summarizes the classes of supply we found to be analogous or clearly non-analogous between HMP (regardless of their actual mass or quantity) relative to a lunar or Martian base. With some understanding of the inherent differences between HMP and planetary bases, the inventory data from HMP can be understood as partially analogous to lunar/Martian exploration bases. The lessons learned from field operations and the complications of logistics in remote environments are also analogous to the difficulties that we must expect on planetary bases. Thus, we view the combined products (data, field observations, and experience) of MIT's research at HMP in 2005 as a good entry point for research in the area of logistics for future planetary bases on the Moon and Mars.

This paper provides a comprehensive comparison between the logistics scenarios at HMP and a potential lunar or Mars base, in order to determine the extent of the analogy between them. When certain straightforward differences are accounted for, the data gathered from HMP can be used to validate and inform planetary base logistics models in support of future human lunar and Mars exploration. 


\section{Acknowledgments}

Matthew Silver at MIT also contributed to the work described in this paper, along with the entire team at HMP during the 2005 season. This work was completed as part of the Interplanetary Supply Chain Management \& Logistics Architectures project financially supported by NASA under contract NNK05OA50C. Prof. Olivier de Weck and Prof. David Simchi-Levi, Massachusetts Institute of Technology, serve as the principal investigators, with Dr. Martin Steele from NASA's Kennedy Space Center as COTR. Co-investigators are Dr. Robert Shishko (JPL), Mr. Joe Parrish (Payload Systems Inc.) and Mr. Andy Evans (United Space Alliance LLC).

\section{References}

1. W.A. Evans, O. de Weck, D. Laufer, S. Shull, "Logistics Lessons Learned in NASA Space Flight." NASA/TP-2006-214203, 2006.

2. C. Taylor, M. Song, D. Klabjan, O. de Weck, D. Simchi-Levi, "Modeling Interplanetary Logistics: A Mathematical Model for Mission Planning." SpaceOps 2006, Rome, Italy, Jun 2006.

3. M. Silver, X. Li, O. de Weck, S. Shull, "Autonomous Logistics Technologies for Space Exploration: Experiment Results and Design Considerations." SpaceOps 2006, Rome, Italy, Jun 2006.

4. S. Shull, E. Gralla, M. Silver, O. de Weck, "Logistics Information Systems for Human Space Exploration: State of the Art and Emerging Technologies." SpaceOps 2006, Rome, Italy, Jun 2006.

5. O. de Weck, D. Simchi-Levi, "Haughton-Mars Project Expedition 2005 Final Report." NASA TP20060214196, 2006.

6. E. Crawley, et al., Draper/MIT Concept Evaluation and Refinement (CE\&R) study, Final Report, September 2005

7. W. Larson, L. Pranke, Human Spaceflight: Mission Analysis and Design. New York: McGraw-Hill, 2000 . 


\section{Appendix A}

Complete list of classes and subclasses of supply.

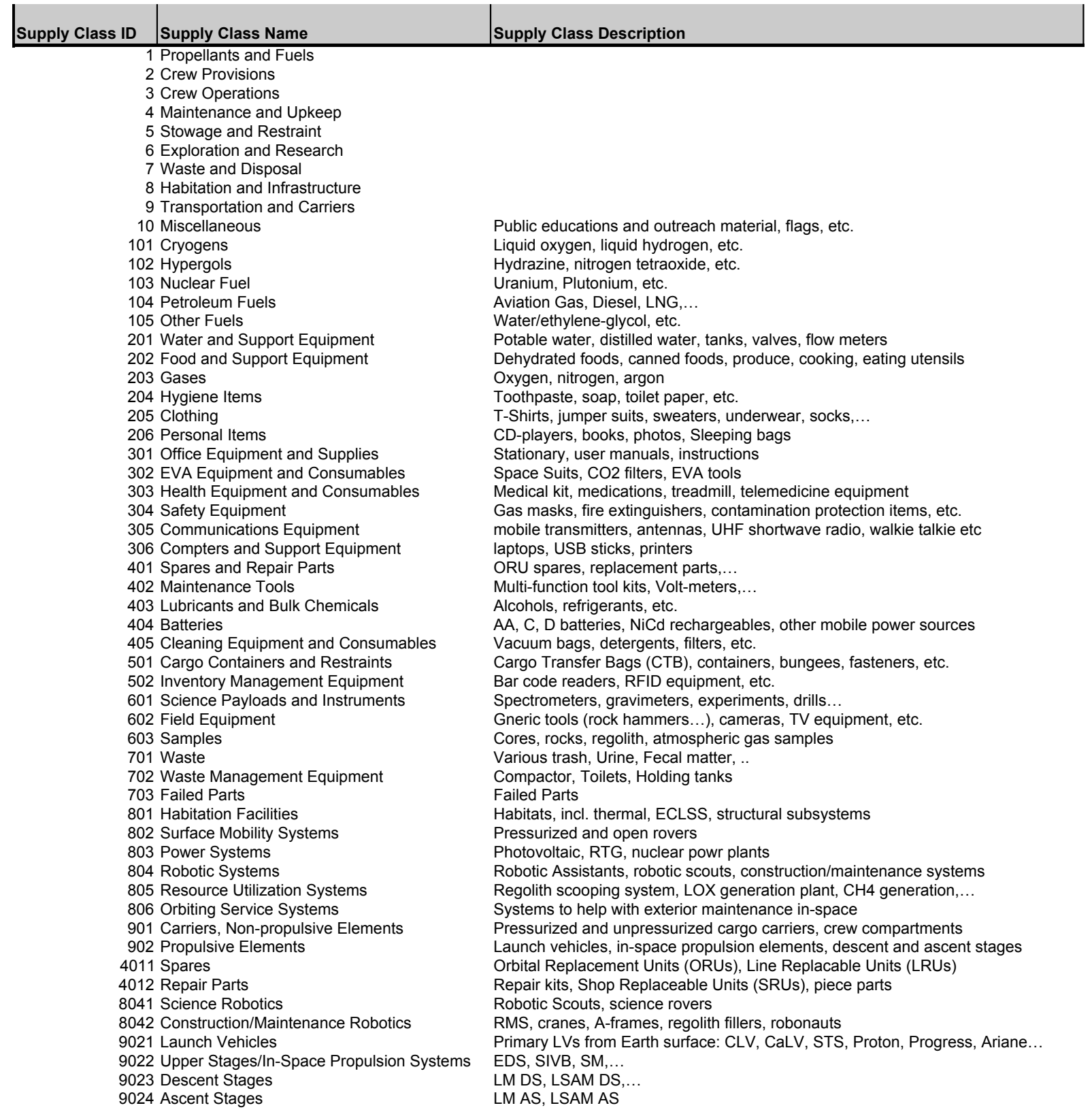

